\title{
INFLUENCIA DEL GÉNERO EN LAS RELACIONES ENTRE INTELIGENCIA EMO- CIONAL, ESTRÉS ACADÉMICO Y SATISFACCIÓN DE LOS ESTUDIANTES
}

\author{
María del Pilar Berrios ${ }^{1}$, Rafael Martos-Montes ${ }^{1}$ y Rafael Martos-Luque ${ }^{2}$ \\ ${ }^{1}$ Universidad de Jaén, España \\ ${ }^{2}$ Emo-é: Inteligencia emocional, España
}

\section{Resumen}

El objetivo de este estudio ha sido analizar el papel del género en la relación entre la Inteligencia Emocional (IE), el estrés académico y la satisfacción con la vida. Para ello, participaron 333 estudiantes de secundaria con edades comprendidas entre los 12 y los 16 años. Los resultados mostraron diferencias estadísticamente significativas en función del género en todas las dimensiones de la IE, en dos de las dimensiones del burnout y en la satisfacción con la vida; siendo las mujeres las que informan de más habilidades para percibir, asimilar y regular las emociones, más desgaste emocional y menos satisfacción con la vida. La relación entre IE y estrés académico puso en evidencia que a mayores niveles de IE menos estrés académico y mayores niveles de satisfacción con la vida. No obstante, tales relaciones estuvieron moduladas por el género: el posible efecto "protector" de la IE sobre el estrés académico es más potente en las chicas, mientras que el posible efecto "beneficioso" de la IE sobre la satisfacción vital en más potente en los chicos. Concluimos que la IE es un recurso personal que protege más a las mujeres que a los hombres del estrés académico, y potencia más la satisfacción con la vida de los hombres que de las mujeres.

Palabras clave: Inteligencia emocional, Estrés, Satisfacción con la vida, Género, Adolescentes.

\section{Abstract}

This research aims to analyze the role of gender in the relationship between Emotional Intelligence (EI), academic stress and life satisfaction. There were involved 333 adolescent students aged between 12 and 16 years. The results showed statistically significant differences according to gender in all the EI dimensions, in two of the burnout dimensions and in life satisfaction; being women those who report more abilities to perceive, assimilate and regulate emotions, more emotional exhaustion and less satisfaction with life. The relationship between EI and academic stress showed that at higher EI levels less academic stress, while at higher EI levels, higher levels of satisfaction with life; However, these relationships were modulated by sex: being a woman enhances the possible "protective" effect of EI on academic stress, while being a man enhances the possible "beneficial" effect of EI on life satisfaction. We conclude that EI is a personal resource that seems to counteract academic stress, more strongly in women than in men, and enhance life satisfaction, to a greater extent in men than in women.

Keywords: Emotional intelligence, Stress, Life satisfaction, Gender, Adolescent.

Correspondencia.

María del Pilar Berrios

rmartos@ujaen.es

Departamento de Psicología. Universidad de Jaén 


\section{Introducción}

En el siglo XXI, el concepto de ser humano nos ha llevado a superar el antiguo debate razón-emoción. En la actualidad, la comunidad científica y psicopedagógica considera que ambas dimensiones actúan conjuntamente en la formación integral de la persona. Aunque la educación se ha centrado fundamentalmente en el plano racional, ignorando completamente el emocional con argumentos como: "ese tema es cuestión de la familia...", "la educación emocional no es prioritaria en el ámbito educativo...", "lo prioritario es que el alumno aprenda...", "ya lo trabajamos transversalmente...", estos argumentos ya no se sostienen. Hoy por hoy no podemos ignorar que las emociones forman parte del ser humano, y la educación emocional debe estar presente y explícitamente incluida en el currículo formal de los alumnos. De hecho, en el informe Delors (1996) se recogen 4 pilares fundamentales de la educación del siglo XXI: (1) aprender a conocer, (2) aprender a hacer, (3) aprender a vivir con los demás y (4) aprender a ser; y las habilidades sociales y emocionales tienen un papel fundamental en todos ellos. En la etapa escolar, especialmente en educación secundaria que es una etapa de muchos cambios sociales, contextuales, y personales, la Inteligencia Emocional (IE) es un recurso personal que, por un lado, previene riesgos importantes como el estrés, el síndrome de burnout, la ansiedad social, la depresión, los pensamientos rumiativos (Extremera y Fernández-Berrocal, 2003; Fernández-Berrocal et al., 2006; Salovey et al., 2002), los comportamientos agresivos (Liau et al., 2003; Petrides et al., 2004) y el consumo de tabaco, alcohol y drogas (Trinidad et al., 2003; Peterson et al., 2011); y por otro, favorece el proceso enseñanza-aprendizaje, el ajuste psicosocial, el bienestar y el rendimiento de los estudiantes (Extremera y Fernández-Berrocal, 2015; Sánchez-Álvarez et al., 2020).

\section{IE y estrés académico}

El estrés académico y el síndrome de burnout han sido objeto de estudio en numerosas ocasiones. Las primeras investigaciones se centraron fundamentalmente en profesiones asistenciales (profesionales de la salud, docentes, trabajadores sociales, policías...) pero el estudio de este síndrome se ha ampliado a otros colectivos, entre ellos el de los estudiantes. El síndrome de burnout en el contexto académico es conceptualizado como el agotamiento o desgaste emocional, el cinismo y la percepción de ineficacia del alumnado sobre las tareas y actividades académicas. Los estudios que han analizado la relación entre IE y el síndrome de burnout en estudiantes, en general han encontrado relaciones negativas entre estas variables. Concretamente se ha comprobado que la IE correlaciona negativamente con el agotamiento emocional y el cinismo, y positivamente con la eficacia académica percibida (Cazan y Nastasa,
2015; Extremera et al., 2007; Usán-Supervía et al., 2020). De acuerdo con Montes-Berges y Augusto-Landa (2007), los estudiantes con mayor claridad emocional identifican mejor las emociones asociadas a las situaciones de estrés, e invierten menos tiempo en atenderlas para usar correctamente sus recursos cognitivos en estrategias de afrontamiento adaptativas. Recientemente, Fiorilli et al. (2020) han encontrado un fuerte efecto indirecto de la IE en el agotamiento escolar a través de la ansiedad y la resiliencia. Esto es, los estudiantes con más IE tenían menos probabilidades de experimentar ansiedad escolar y eran más resilientes lo que, a su vez, redujo el riesgo de agotamiento escolar.

\section{IE y satisfacción con la vida}

La satisfacción con la vida refleja el bienestar subjetivo personal o el grado en que un individuo valora de forma favorable su calidad de vida (Veenhoven, 1996). Ha sido considerado como el componente cognitivo del bienestar subjetivo, y la evidencia empírica indica que se relaciona con indicadores de funcionamiento adaptativo en la infancia y adolescencia (Huebner, 2004).

Existen numerosos estudios que han abordado la relación entre IE y satisfacción con la vida, cuyos resultados indican que los estudiantes con más IE informan de mayores niveles de satisfacción vital y de bienestar subjetivo (Balluerka et al., 2016; Cazan y Nastasa, 2015; Extremera y Fernández -Berrocal, 2005; Gallagher y Vella-Brodrick, 2008; Kong et al. 2012; Palmer et al., 2002; Petrides et al., 2007; Riaz et al., 2009; Saklofske et al., 2003; Sánchez-Álvarez et al., 2016). Por otra parte, también se ha comprobado que las relaciones entre la IE y la satisfacción vital dependen de los niveles de estrés académico. De forma que, los alumnos pre -universitarios con altos niveles de estrés percibido y alta claridad emocional están más satisfechos con su vida que los que tienen menos claridad emocional. Sin embargo, cuando los niveles de estrés percibido son bajos, no hay diferencias en la satisfacción con la vida entre los estudiantes de alta y baja claridad emocional (Extremera et al., 2009). En otro reciente estudio con alumnos de secundaria se ha comprobado que la reparación emocional ejerce una influencia directa e indirecta, a través de la resiliencia, en la satisfacción con la vida. Por lo tanto, esta habilidad emocional contribuye a superar las adversidades y a experimentar mayor satisfacción vital (Ramos-Díaz et al., 2019). Lopez-Zafra et al. (2019) afirman que los alumnos con más Inteligencia Emocional Percibida (IEP) tienen más apoyo social de su familia, amigos y centro educativo, lo que a su vez influye positivamente en la satisfacción con su vida y negativamente en sus niveles de depresión. 


\section{Diferencias de género en IE, burnout y satisfacción con la vida}

Cuando se han analizado las diferencias de género en IE, los resultados son contradictorios y dispares, dependiendo de la forma en que se evalúe la IE. En los estudios que han utilizado medidas de auto-informe contamos con diferentes resultados. Por ejemplo, algunos autores no han encontrado diferencias claras entre hombres y mujeres (Brackett et al., 2006; Harrod y Scheer, 2005; Salavera, et al., 2017; Uçak y Yildirim, 2020); mientras que en otros trabajos se comprueba que las mujeres informan de mayores habilidades emocionales que los hombres (Austin et al., 2005; Brackett, et al., 2004; Pandey y Tripathi, 2004; Silvery et al., 2004, Van Rooy et al., 2005). Por otra parte, cuando la IE es evaluada mediante pruebas de ejecución, donde se plantean diferentes dilemas emocionales cuya resolución requiere el uso de habilidades socio-emocionales, las mujeres obtienen mejores resultados que los hombres (Ciarrochi et al., 2000; Extremera et al., 2006; Palmer et al., 2005; Valadez et al, 2013). En el estudio de Cabello et al. (2016) se hallaron diferencias en función del género en adultos con edades comprendidas entre los 17 y los 76 años, siendo las mujeres las que obtuvieron mayores puntuaciones tanto en la IE global como en cada una de las dimensiones que la componen. Estos resultados son complementarios a los de Fernández-Berrocal et al. (2012), que comprobaron que estas diferencias se atenúan con la edad.

En las investigaciones sobre las diferencias de género en el síndrome de burnout también se obtienen resultados contradictorios. En los estudios llevados a cabo con estudiantes universitarios, si bien en algunos casos se confirma que las mujeres registran mayores niveles de burnout en las tres dimensiones que lo forman (agotamiento emocional, cinismo y falta de eficacia académica) (Rico, 2009; RodríguezVillalobos et al., 2019) y utilizan estrategias de afrontamiento diferentes a las de los hombres (Conti et al., 2018; Matud, 2004); en otros trabajos se encuentra que son los hombres los que obtienen puntuaciones más altas en la escala de cinismo (Martínez y Salanova, 2003). Por otra parte, en la investigación de Galán et al., (2011) no se encuentran diferencias en el síndrome de burnout en función del género. A pesar de estos resultados contradictorios, Purvanova y Muros (2010), tras realizar un meta-análisis en el que revisaron 183 estudios sobre burnout, concluyen que existen diferencias en las dimensiones de este síndrome según el género, siendo las mujeres más propensas a puntuar alto en la dimensión agotamiento emocional, mientras que los hombres registran mayores niveles de despersonalización.

Por último, cuando se han abordado las diferencias de género en la satisfacción vital, los resultados favorecen ligeramente a los varones (Goldbeck et al., 2007), aunque tam- bién existen estudios que no hallan diferencias (Ash y Huebner, 2001; Gilman y Huebner, 2003; Huebner et al., 2000). No obstante, Pinquart y Sorensen (2001), realizaron un meta-análisis de 300 estudios empíricos y encontraron diferencias pequeñas pero significativas según las cuales los hombres presentan mayores niveles de satisfacción que en las mujeres.

Partiendo de los resultados de las investigaciones previas que han analizado la relación entre IE, género, síndrome de burnout y satisfacción con la vida, los objetivos de este estudio y sus respectivas hipótesis asociadas, son los siguientes:

Comprobar si existen diferencias en IE, burnout y satisfacción con la vida de los estudiantes en función del género.

H1. Las mujeres mostrarán más IE, mayores niveles de burnout y menos satisfacción con la vida que los hombres.

Analizar si la IE de los estudiantes se relaciona con sus niveles de burnout y satisfacción con la vida.

H2. La IE de los estudiantes estará asociada a menores niveles de burnout y mayores niveles de satisfacción vital.

Comprobar si la relación entre la IE, el estrés académico y la satisfacción con la vida de los estudiantes depende del género.

H3. El género moderará las relaciones entre IE, burnout y satisfacción con la vida.

\section{Metodología}

\section{Participantes}

La muestra estuvo compuesta por 333 estudiantes (141 mujeres y 192 hombres) de Educación Secundaria Obligatoria (ESO) con edades comprendidas entre los 12 y los 16 años $(M=13,43$ años; $D T=1,25)$. El $39 \%$ de los participantes cursaba primero de la ESO, el $22 \%$ de segundo, el $8 \%$ de tercero, y el $31 \%$ restante estaba en cuarto de la ESO. Estos alumnos pertenecían a diferentes centros del ámbito rural y urbano de Jaén capital y provincia, y fueron seleccionados a través de un muestreo no probabilístico de conveniencia.

\section{Instrumentos de medida}

Wong and Law's Emotional Intelligence Scale (WLEIS; Wong y Law, 2002; validación española de Extremera et al., 2019). Esta prueba de 16 ítems con formato de respuesta tipo Likert de 1 a 7 (1= completamente en desacuerdo, $7=$ completamente de acuerdo) evalúa cuatro dimensiones de la Inteligencia Emocional Percibida (IEP): percepción emocional intrapersonal, percepción emocional interpersonal, asimilación emocional, y regulación emocional. Las dimensiones percepción emocional intra e interpersonal, se refieren a la habilidad de percibir las emociones propias y 
las de los demás, respectivamente (p.ej., "La mayoría de las veces sé distinguir por qué tengo ciertos sentimientos", "Soy un buen observador de las emociones de los demás). La dimensión asimilación emocional se define como la habilidad para utilizar las emociones de manera que faciliten el pensamiento y el comportamiento para el logro de objetivos (p.ej., "Soy una persona auto-motivadora"). La dimensión regulación emocional hace referencia a la habilidad de manejar las emociones (p.ej., "Me puedo calmar fácilmente cuando me siento enfadado"). Esta escala está basada en el modelo teórico de Mayer y Salovey (1997), y presenta buenas propiedades psicométricas con un coeficiente alpha de Cronbach de 0,91 para la escala total, y con coeficientes que oscilan entre 0,79 y 0,84 en las cuatro subescalas (Extremera et al., 2019).

Maslach Burnout Inventory-Student Survey (MBI-SS; Schaufeli et al, 2002; validación española de Pérez-Fuentes et al., 2020). Se trata de un cuestionario formado por 16 ítems con formato de respuesta tipo Likert de 0 a 6 puntos $(0=$ nunca, 6 = siempre) que evalúa el síndrome de burnout en el ámbito académico (estrés académico), el cual está formado por tres dimensiones: agotamiento emocional (p.ej., "Estoy emocionalmente agotado por mis estudios"), pérdida de interés por el estudio (cinismo) (p.ej., "Me he vuelto más cínico respecto a la utilidad de mis estudios"), y creencias sobre la propia capacidad para realizar el trabajo académico (autoeficacia académica) (p.ej., "Creo que contribuyo efectivamente durante las clases en el instituto"). Sus propiedades psicométricas indican un coeficiente alpha de Cronbach de 0,75 para el agotamiento emocional, 0,83 para el cinismo, y 0,82 para la autoeficacia académica (Pérez-Fuentes et al., 2020).

The Satisfaction With Life Scale (SWLS; Diener et al., 1985; validación española de Atienza et al., 2000; 2003). Esta escala evalúa cómo de satisfechas están las personas con su propia vida, está formada por 5 ítems con formato de respuesta tipo Likert de 7 puntos $(1=$ totalmente en desacuerdo, $7=$ totalmente de acuerdo) (p.ej., "En la mayoría de las cosas, mi vida está cerca de mi ideal, "Hasta ahora, he conseguido las cosas que para mí son importantes en la vida"), y presenta una buena consistencia interna con un coeficiente alpha de Cronbach de 0,84 (Atienza et al., 2000).

\section{Diseño y procedimiento}

Este trabajo es un estudio correlacional transversal en el que, una vez seleccionadas las muestras en cada centro educativo, y obtenidos los permisos y consentimientos informados correspondientes, se administraron los diferentes instrumentos de medida de manera colectiva. El Comité de ética de la Universidad de Jaén y los departamentos de orientación de los centros de secundaria autorizaron el desarrollo del estudio, la participación fue voluntaria y los padres y/o tutores fueron informados y dieron su consentimiento. Los datos recogidos fueron procesados y analizados mediante el programa estadístico IBM SPSS v. 24. En todos los análisis estadísticos, se trabajó con un nivel de significación $p \leq 0,05$ y un nivel de confianza del 95\%.

\section{Resultados}

\section{Análisis descriptivos y comparativos}

En la Tabla 1 se muestran los análisis descriptivos de las variables objeto de estudio y los resultados de la prueba de contraste de medias $t$ de Student. Como puede observarse, existen diferencias estadísticamente significativas en función del género en todas las dimensiones de IE, en dos de las dimensiones del burnout y en la satisfacción con la vida; siendo las mujeres las que informan de más habilidades para percibir, asimilar y regular las emociones, más desgaste emocional y menos satisfacción con la vida.

Tabla 1.

Estadísticos descriptivos y t de Student para el contraste de diferencias de género

\begin{tabular}{|c|c|c|c|c|c|c|c|c|}
\hline & \multicolumn{2}{|c|}{$\begin{array}{c}\text { Muestra total } \\
(\mathbf{n}=333)\end{array}$} & \multicolumn{2}{|c|}{ Mujeres $(n=141)$} & \multicolumn{2}{|c|}{ Hombres $(n=192)$} & \multirow[b]{2}{*}{$t$-Student } & \multirow[b]{2}{*}{$p$} \\
\hline & Media & DT & Media & DT & Media & DT & & \\
\hline $\begin{array}{l}\text { Percepción } \\
\text { emocional } \\
\text { intrapersonal }\end{array}$ & 5,33 & 1,08 & 5,44 & 1,04 & 5,18 & 1,12 & 2,19 & 0,029 \\
\hline $\begin{array}{l}\text { Percepción } \\
\text { emocional } \\
\text { interpersonal }\end{array}$ & 5,15 & 1,00 & 5,37 & 0,88 & 4,99 & 1,06 & $-3,49$ & 0,001 \\
\hline $\begin{array}{l}\text { A simil ación } \\
\text { emocional }\end{array}$ & 5,05 & 1,27 & 5,16 & 1,32 & 4,89 & 1,19 & 1,84 & 0,050 \\
\hline $\begin{array}{l}\text { Regulación } \\
\text { emocional }\end{array}$ & 4,42 & 1,34 & 4,63 & 1,33 & 4,13 & 1,30 & 3,43 & 0,001 \\
\hline IE $P$ total & 4,97 & 0,85 & 5,04 & 0,88 & 4,88 & 0,79 & 1,92 & 0,050 \\
\hline $\begin{array}{l}\text { Desgaste } \\
\text { emocional }\end{array}$ & 4,85 & 1,01 & 4,96 & 1,06 & 4,70 & 0,92 & 2,37 & 0,018 \\
\hline Cinismo & 3,23 & 1,46 & 3,35 & 1,46 & 3,07 & 1,45 & 1,74 & 0,082 \\
\hline $\begin{array}{l}\text { A utoefi caci a } \\
\text { académica }\end{array}$ & 2,81 & 1,03 & 2,54 & 1,03 & 3,00 & 0,96 & 4,12 & 0,000 \\
\hline $\begin{array}{l}\text { Satisfacción } \\
\text { conlavida }\end{array}$ & 5,14 & 1,27 & 4,98 & 1,30 & 5,26 & 1,21 & 1,98 & 0,048 \\
\hline
\end{tabular}

Nota. DT= Desviación Típica $;$ IEP= Inteligencia Emocional Percibida. 


\section{Análisis de correlación}

Con el fin de examinar las relaciones entre la IEP, el síndrome de burnout y la satisfacción con la vida en función del género, se llevó a cabo un análisis de correlación de Pearson para la muestra dividida entre hombres y mujeres. En la Tabla 2 se presentan los resultados de estas correlaciones por género. Los resultados indican que existen asociaciones negativas significativas entre la IEP y dos dimensiones del burnout (agotamiento emocional y cinismo) y asociaciones positivas significativas entre IEP y satisfac- ción con la vida en todos los casos, esto es, tanto para los hombres como para las mujeres. Se llevó a cabo un análisis de transformación $r-z$ de Fisher para examinar si existían diferencias entre los coeficientes por género. Los resultados indicaron que las correlaciones entre IEP y desgaste emocional eran significativamente más altas en las mujeres que en los hombres $(z=-2,32 ; p<0,01)$; y las correlaciones entre IEP y satisfacción vital eran significativamente mayores para los hombres que para las mujeres $(z=2,18$; $p<$ $0,05)$.

Tabla 2.

Correlaciones entre las variables de interés para hombres y mujeres

\begin{tabular}{llllll}
\hline & 1 & 2 & 3 & 4 & 5 \\
\hline 1. IEP total & - & $-0,561^{* *}$ & $-0,216^{* *}$ & 0,140 & $0,649^{* *}$ \\
2. A gotamiento emocional & $-0,701^{* *}$ & - & 0,107 & $-0,040$ & $-0,810^{* *}$ \\
3. Cini smo & $-0,212^{* *}$ & 0,010 & - & $-0,473^{* *}$ & $-0,328^{* *}$ \\
4. Autoeficacia académica & 0,032 & 0,104 & $0,509^{* *}$ & - & $0,211^{*}$ \\
5. Sati sfacción vital & $0,447^{* *}$ & $-0,836^{* *}$ & $-0,194^{* *}$ & $-0,060$ & - \\
\hline
\end{tabular}

Nota. IEP= Inteligencia Emocional percibida. Las correlaciones por debajo de la diagonal son para mujeres $(N=141)$, y las correlaciones por encima de la diagonal son para hombres $(N=192) ;{ }^{*} p<0,05 ; * * p<0,01$

Tabla 3.

Resultados del análisis de regresión jerárquica moderada para los síntomas del burnout y la satisfacción con la vida

\begin{tabular}{|c|c|c|c|c|c|c|c|c|c|c|c|c|c|c|c|c|}
\hline \multirow[t]{2}{*}{ Predictor } & \multicolumn{4}{|c|}{ Agotamientoemociona } & \multicolumn{4}{|c|}{ Cinismo } & \multicolumn{4}{|c|}{ A utoefi cadia académica } & \multicolumn{4}{|c|}{ Satisfación œon la vida } \\
\hline & $R^{2}$ & $F$ & $\boldsymbol{\beta}$ & $\Delta R^{2}$ & $R^{2}$ & $F$ & $\boldsymbol{\beta}$ & $\Delta R^{R}$ & $R^{2}$ & $F$ & $\boldsymbol{\beta}$ & $\Delta R^{2}$ & $R^{2}$ & $F$ & $\boldsymbol{\beta}$ & $\Delta R^{2}$ \\
\hline Paso 1 & 0,003 & 1,05 & & 0,003 & 0,000 & 0,08 & & 0,000 & 0,004 & 1,22 & & 0,004 & 0,001 & 0,25 & & 0,001 \\
\hline Edad & & & 0,07 & & & & 0,023 & & & & 0,08 & & & & 0,042 & \\
\hline Paso 2 & 0,432 & 125,63 & & $0,429^{* *}$ & 0,041 & 7,05 & & 0,041 & 0,004 & 0,62 & & 0,000 & 0,334 & 82,61 & & $0,333^{\cdots}$ \\
\hline IEP & & & $-0,86^{\cdots \cdots}$ & & & & $-0,181$ & & & & 0,19 & & & & $0,816^{\cdots}$ & \\
\hline Paso 3 & 0,438 & 65,37 & & 0,006 & 0,055 & 6,34 & & 0,014 & 0,057 & 0,63 & & $0,053^{\cdots}$ & 0,337 & 55,76 & & 0,003 \\
\hline Género & & & 0,08 & & & & $-0,118$ & & & & $-023^{\cdots}$ & & & & $-0,062$ & \\
\hline Paso 4 & 0,464 & 85,45 & & $0,016^{\circ}$ & 0,055 & 4,75 & & 0,000 & 0,063 & 5,53 & & 0,006 & 0,354 & 43,17 & & $0,017^{\circ}$ \\
\hline IEPXGénero & & & $0,25^{\circ}$ & & & & $-0,033$ & & & & $-0,24$ & & & & $0,260^{\circ}$ & \\
\hline
\end{tabular}

Nota: IEP = Inteligencia Emocional Percibida Loscoeficientesbeta representadosson losestandarizadosparala ecuaciónfinal (paso 4); " $p<0,05$; ${ }^{* *} p<0,01 ;{ }^{* * *} p<0,001$ 


\section{Análisis de Regresión Jerárquica Moderada}

Con el fin de examinar el efecto moderador del género en la asociación entre la IEP, el síndrome de burnout y la satisfacción con la vida, se llevó a cabo un análisis de regresión jerárquica. En dicho análisis, se incluyeron como variables dependientes cada una de las dimensiones del burnout (agotamiento emocional, cinismo y autoeficacia académica) y la satisfacción vital. En el primer paso se introdujo la edad como variable control, en el segundo se incluyó la IEP y en el tercero la variable género. En el paso 4 se añadieron las interacciones entre IEP y género siguiendo el procedimiento estándar de centrar las medias de las variables para examinar los efectos de moderación (Aiken y West, 1991). De forma complementaria, tras el análisis de regresión se utilizó el paquete macro PROCESS (Hayes, 2013) para conocer los efectos de interacción entre las variables.

Como puede observarse en la Tabla 3 , el $43 \%$ de la varianza total del agotamiento emocional fue explicada por la IEP $\left[F(6,332)=125,63 ; p<0,01, R^{2}=0,432\right]$. La variable IEP resultó predictora del agotamiento emocional en la muestra total $[\beta=-0,863, t(332)=6,92 ; p<0,000]$. El modelo también fue significativo para la interacción entre IEP y género $[\beta=0,250, t(332)=3,59 ; p<0,001]$. El efecto de la IEP sobre el cansancio emocional mostró una relación más intensa en las mujeres $(\beta=-0,72 ; p<0,001)$ que en los hombres $(\beta=-0,38 ; p<0,01)$.

Por otra parte, la IEP no explica un porcentaje significativo de varianza ni del cinismo ni de la autoeficacia académica $\left[F(6,332)=7,05 ; p>0,05, R^{2}=0,041\right.$, y $F(6,332)=$ 0,$62 ; p>0,05, R^{2}=0,000$, respectivamente]. El modelo tampoco fue significativo para la interacción entre IEP y género en ninguno de los dos casos $[\beta=0,102, t$ (332) $=$ 2,$01 ; p>0,05, \mathrm{y} \beta=0,085, t(332)=1,69 ; p>0,05$, respectivamente].

Por último, el 33,3\% de la varianza total de la satisfacción vital fue explicada por la IEP $[F(6,332)=82,61 ; p<$ $\left.0,001, R^{2}=0,334\right]$. La variable IEP resultó predictora de la satisfacción vital en la muestra total $[\beta=0,816, t$ (332) $=$ $6,03 ; p<0,000]$. El modelo fue significativo para la interacción entre IEP y género $[\beta=0,238, t(332)=2,89 ; p<$ $0,001]$. El efecto de la IEP sobre la satisfacción vital mostró una relación más intensa en los hombres $(\beta=0,95 ; p<$ $0,001)$ que en las mujeres $(\beta=0,68 ; p<0,01)$.

\section{Discusión y conclusiones}

Este trabajo tuvo como principales objetivos: 1) analizar si existen diferencias entre los chicos y las chicas de educación secundaria en la IEP, el síndrome de burnout y la satisfacción con la vida, 2) comprobar si la IEP de los estudiantes se asocia negativamente con sus niveles de burnout y positivamente con su satisfacción vital, y 3) estudiar si las relaciones entre estas variables dependen del género. Los principales resultados indicaron que existen diferencias estadísticamente significativas entre las alumnas y los alumnos de la ESO en las variables objeto de estudio. Concretamente, las chicas presentan mayores niveles de IEP, y más desgaste emocional y cinismo que los chicos; y ellos registran menores niveles de IEP, y puntuaciones más altas en autoeficacia académica y satisfacción con la vida. Estos resultados confirman la $\mathrm{H} 1$, y son consistentes con los de otros estudios previos que han mostrado diferencias entre hombres y mujeres en IEP (Pena et al., 2012; Whitmann et al., 2009), en el síndrome de burnout (Purvanova y Muros, 2010) y en la satisfacción vital (Pinquart y Sorensen, 2001). Estas diferencias pueden deberse a los patrones de socialización que mantienen estereotipos culturales de género interiorizados por jóvenes y adolescentes que favorecen diferencias entre hombres y mujeres en la expresión y manejo de las emociones, el establecimiento de objetivos y la orientación hacia el logro, entre otros (Colás y Villaciervos, 2007).

Por otra parte, también hemos encontrado que, en todos los casos (tanto en alumnas como en alumnos) la IEP se relaciona negativamente con las dimensiones tóxicas del burnout (desgaste emocional y cinismo), y positivamente con la satisfacción vital, siendo estas relaciones más fuertes en las chicas que en los chicos. Estos resultados confirman la $\mathrm{H} 2$, y coinciden con los de otros autores según los cuales la IEP, por una parte, amortigua los efectos del estrés académico y contribuye a afrontar desafíos (Saklofske et al., 2012; Schneider et al., 2013), y por otra, facilita el bienestar (Eryilmaz, 2011) y la satisfacción con la vida (Extremera y Rey, 2016).

Por último, nuestros resultados indican que la IEP explica el $43 \%$ del desgaste emocional y el $33,3 \%$ de la satisfacción vital de los estudiantes, y que las relaciones entre estas variables dependen del género. Estos resultados confirman la $\mathrm{H} 3$, ya que la relación entre la IEP y el agotamiento emocional es más negativa en las chicas que en los chicos, y la relación entre la IEP y la satisfacción vital es más positiva en los chicos que en las chicas. A lo largo de este trabajo hemos puesto de manifiesto que, de acuerdo con la evidencia empírica disponible, podemos afirmar que la IE es un recurso personal que juega un doble rol en la vida de las personas: como amortiguador del estrés y como facilitador del bienestar. Según nuestros resultados, a las chicas adolescentes este recurso personal les sirve fundamentalmente para amortiguar uno de los efectos más nocivos del estrés académico, esto es, el agotamiento emocional; mientras que a los chicos les sirve sobre todo para incrementar sus niveles de bienestar, concretamente, la satisfacción con la vida. Estas diferencias por género pueden deberse a que las chicas y los chicos adolescentes habitan en mundos emocionales diferentes y utilizan distintas estrategias de 
regulación emocional para conseguir sus propios objetivos o prioridades (Shields, 2002; Salguero et al., 2015). En cualquier caso, el desarrollo de la IE beneficia de una forma u otra a todos los estudiantes, a las chicas les beneficia especialmente en el afrontamiento del estrés, y a los chicos en el incremento de uno de los indicadores del bienestar (satisfacción vital). Puesto que a través de la práctica y el entrenamiento se pueden perfeccionar las habilidades socioemocionales en diferentes situaciones de la vida cotidiana (Fernández-Berrocal y Extremera, 2005; Mayer et al., 2008), se justifica la necesidad de llevar a cabo en los centros educativos programas de entrenamiento estructurados en sesiones secuenciales, en las que se utilicen formas activas de aprendizaje para desarrollar gradualmente habilidades socio-emocionales concretas (p.ej., percibir adecuadamente las emociones propias y las de los demás, usar la emociones para tomar decisiones acertadas de acuerdo con los objetivos de la persona, comprender las emociones propias y las de los demás, y regular la emociones propias y las de los demás; para una revisión más amplia véase Mayer y Salovey, 1997; Mayer et al., 2008).

Existen diferentes argumentos teóricos que pueden explicar los mecanismos a través de los cuales la IE favorece el funcionamiento académico óptimo. Según Brackett et al. (2005) la IE favorece la calidad de las relaciones sociales, y tener una mejor red social en el ámbito académico facilitará un entorno social adecuado para trabajar y aprender en equipo, obtener mayor apoyo de los compañeros (Hogan et al., 2010) y mejores relaciones con los profesores (Di Fabio y Kenny, 2015). A su vez, las buenas relaciones con los profesores y compañeros pueden proteger a los estudiantes de los efectos negativos del estrés académico, y contribuir a que estén más satisfechos con su vida. Este mecanismo explicativo específico para los estudiantes con alta IE está en consonancia con teorías macro que explican el proceso general del estrés (modelo de las Demandas-Control de Karasek y Theorell, 1990), y el proceso general del bienestar (modelo PERMA de Seligman, 2018; modelo del Bienestar Psicológico de Ryff, 1989) considerando al apoyo social y/o las relaciones sociales positivas como un factor clave en ambos procesos ya que amortigua el estrés, y favorece la satisfacción y el bienestar.

Este estudio presenta algunas limitaciones. Por ello, los resultados que hemos obtenido han de ser considerados con cautela y deben tenerse en cuenta como punto de partida en futuras investigaciones. En primer lugar, al tratarse de un estudio transversal no podemos conocer el impacto de la IE a lo largo del tiempo. Para ello, sería necesario llevar a cabo estudios longitudinales en hombres y mujeres con el fin de comprobar si el impacto diferencial en función del género que hemos encontrado en uno de los momentos más delicados de la vida académica (educación secundaria) se mantiene a lo largo del tiempo, o por el contrario en niveles más avanzados de educación dicho impacto se iguala en toda la población. Además, en este trabajo no hemos examinado el posible efecto del apoyo social y de los rasgos de personalidad en la relación entre IEP, género, síndrome de burnout y satisfacción con la vida. Si bien, el apoyo social se ha contemplado como uno de los mecanismos a través de los cuales la IE amortigua el estrés académico y favorece la satisfacción vital, sería necesario analizar los efectos de la interacción IE x apoyo social en los estudiantes de la ESO. Por último, también sería muy interesante comprobar si se replican nuestros resultados utilizando medidas objetivas de IE, ya que en recientes meta-análisis se ha puesto de manifiesto que los efectos de la IE en el rendimiento académico, la salud o el bienestar pueden variar en función del tipo de medida utilizada para evaluarla (Martins et al., 2010; Sánchez-Álvarez et al., 2016; 2020).

A pesar de estas limitaciones, los resultados de esta investigación pueden ayudar a entender mejor el papel diferencial del género en la relación de la IE con menores niveles de desgaste emocional y mayores niveles de satisfacción vital en estudiantes de secundaria. Las diferencias de género que hemos encontrado en el impacto de la IE sobre el ajuste psicológico de los estudiantes aportan evidencias a tener en cuenta en el diseño de programas de entrenamiento de IE diferenciales para hombres y mujeres (Gutiérrez-Moret et al., 2016).

En conclusión, la educación emocional en los centros educativos constituye uno de los retos más importantes de nuestra sociedad por su vinculación con entornos de aprendizaje más favorables y claros beneficios para el alumnado (Vesely et al., 2013), y la implementación de la perspectiva de género en los programas de intervención parece inexorable.

\section{Referencias}

Aiken, L. y West, S.G. (1991). Multiple regression: Testing and Interpreting Interactions. Sage.

Ash, C. y Huebner, E.S. (2001). Environmental events and life satisfaction reports of adolescents: A Test of Cognitive Mediation. School Psychology International, 22, 320-336. https://doi.org/10.1177\% 2F0143034301223008

Atienza, F.L., Pons, D., Balaguer, I. y García-Merita, M.L. (2000). Propiedades psicométricas de la Escala de Satisfacción con la Vida en adolescentes. Psicothema, 12(2), 331-336.

Atienza, F.L., Balaguer, I. y García-Merita, M.L. (2003). Satisfaction with Life Scale: Analysis of factorial invariante across sexes. Personality and Individual Differences, 35, 1255-1260. https://doi.org/10.1016/ S0191-8869(02)00332-X 
Austin, E.J., Evans, P., Goldwater, R. y Potter, V. (2005). A preliminary study of emotional intelligence, empathy and exam performance in first year medical students. Personality and Individual Differences, 39 (8), 1395-1405. https://doi.org/10.1016/ j.paid.2005.04.014

Balluerka, N., Gorostiaga, A., Alonso-Arbiol, I. y Aritzeta, A. (2016). Peer attachment and class emotional intelligence as predictors of adolescents' psychological well-being: A multilevel approach. Journal of Adolescence, 53, 1-9. https://doi.org/10.1016/ j.adolescence.2016.08.009

Brackett, M.A., Mayer, J.D. y Warner, R. (2004). Emotional intelligence and its relation to everyday. Personality and Individual Differences, 36, 1387-1402. https://doi.org/10.1016/S0191-8869(03)00236-8

Brackett, M., Warner, R.M. y Bosco, J.S. (2005). Emotional intelligence and relationship quality among couples. Personal Relationships, 12(2), 197-212. https://doi.org/10.1111/j.1350-4126.2005.00111.x

Brackett, M.A., Rivers, S.E., Shiffman, S., Lerner, N. y Salovey, P. (2006). Relating emotional abilities to social functioning: A comparison of self-report and performance measures of emotional intelligence. Journal of Personality and Social Psychology, 91 (4), 780-795. https://doi.org/10.1037/00223514.91.4.780

Cabello, R., Sorrel, M.A., Fernández-Pinto, I., Extremera, N. y Fernández-Berrocal, P. (2016). Age and gender differences in ability emotional intelligence in adults: A cross-sectional study. Developmental Psychology, 52(9), 1486-1492. http:// dx.doi.org/10.1037/dev0000191

Cazan, A.M. y Nastasa, L.E. (2015). Emotional intelligence, satisfaction with life and burnout among university students. Procedia - Social and Behavioral Sciences 180, 1574-1578. https://doi.org/10.1016/ j.sbspro.2015.02.309

Ciarrochi, J.V., Chan, A.Y.C. y Caputi, P. (2000). A critical evaluation of the emotional intelligence construct. Personality and Individual Differences, 28 (3), 539-561. https://doi.org/10.1016/S0191-8869(99) 00119-1

Colás, P. y Villaciervos, P. (2007). La interiorización de los estereotipos de género en jóvenes y adolescentes. Revista de Investigación Educativa, 25(1), 35-38. Recuperado de https://revistas.um.es/rie/article/ view/96421

Conti, J.V., Muntaner, A. y Sampol, P.P. (2018). Diferen- cias de estrés y afrontamiento del mismo según el género y cómo afecta al rendimiento académico en estudiantes universitarios. Contextos Educativos: Revista de Educación, (22), 181-195. http:// doi.org/10.18172/con.3369

Delors, J. (1996). La educación encierra un tesoro. Informe de la Comisión Internacional sobre la Educación para el siglo XXI. Recuperado de http:// www.unesco.org/education/pdf/DELORS_S. PDF.

Di Fabio, A. y Kenny, M.E. (2015). The contributions of emotional intelligence and social support for adaptive career progress among Italian youth. Journal of Career Development, 42(1), 48-59. https:// doi.org/10.1177/0894845314533420

Diener, E., Emmons, R., Larsen, R.J. y Griffin, S. (1985). The satisfaction with Life Scale. Journal of Personality Assessment, 49, 71-75. https:// doi.org/10.1207/s15327752jpa4901_13

Eryllmaz, A. (2011). A model of subjective well-being for adolescents in high school. Journal of Happiness Studies, 13(2), 275-289. https://doi.org/10.1007/ s10902-011-9263-9

Extremera, N., Durán, A. y Rey, L. (2007). Inteligencia emocional y su relación con los niveles de burnout, engagement y estrés en estudiantes universitarios. Revista de Educación, 342, 239-256. Recuperado de http://emotional.intelligence.uma.es/documentos/ pdf50niveles_burnout.pdf

Extremera, N., Durán, A. y Rey, L. (2009). The moderating effect of trait meta-mood and perceived stress on life satisfaction. Personality and Individual Differences, 47(2), 116-121. http://dx.doi.org/10.1016/ j.paid.2009.02.007

Extremera, N. y Fernández-Berrocal, P. (2003). La inteligencia emocional en el contexto educativo: hallazgos científicos de sus efectos en el aula. Revista de Educación, 332(12), 97-116.

Extremera, N. y Fernández-Berrocal, P. (2005). Perceived emotional intelligence and life satisfaction: Predictive and incremental validity using the Trait MetaMood Scale. Personality and Individual Differences, 39, 937-948. https://doi.org/10.1016/ j.paid.2005.03.012

Extremera, N. y Fernández-Berrocal, P. (2015). Inteligencia Emocional y Educación. Grupo 5.

Extremera, N., Fernández-Berrocal, P. y Salovey, P. (2006). Spanish version of the Mayer- SaloveyCaruso Emotional Intelligence Test (MSCEIT) Version 2.0: Reliabilities, age, and gender differences. 
Psicothema, 18, 42-48. Recuperado de http:// www.redalyc.org/articulo.oa? $\mathrm{id}=72709506$

Extremera, N. y Rey, L. (2016). Ability emotional intelligence and life satisfaction: Positive and negative affect as mediators. Personality and Individual Differences, 102, 98-101. https://doi.org/10.1016/ j.paid.2016.06.051

Extremera, N., Rey, L. y Sánchez-Álvarez, N. (2019). Validation of the Spanish version of the Wong Law Emotional Intelligence Scale (WLEIS-S). Psicothema, 31(1), 94-100. https://doi.org/10.7334/ psicothema2018.147

Fernández-Berrocal, P., Alcaide, R., Extremera, N. y Pizarro, D.A. (2006). The role of emotional intelligence in anxiety and depression among adolescents. Individual Differences Research, 4, 16-27.

Fernández-Berrocal, P., Cabello, R, Castillo, R. y Extremera, N. (2012). Gender differences in emotional intelligence: The mediating effect of age. Psicología Conductual, 20(1), 77-89.

Fernández-Berrocal, P. y Extremera, N. (2005). La Inteligencia Emocional y la educación de las emociones desde el modelo de Mayer y Salovey. Revista Interuniversitaria de Formación del profesorado, 19(3), 63-93. Recuperado de https://www.redalyc.org/ articulo.oa? id=274/27411927005

Fiorilli, C., Farina, E., Buonomo, I., Costa, S., Romano, L., Larcan, R. y Petrides, K.V. (2020). Trait emotional intelligence and school burnout: The mediating role of resilience and academic anxiety in high school. International Journal of Environmental Research and Public Health, 17(9), 3058. http:// dx.doi.org/10.3390/ijerph17093058

Galán, F., Sanmartín, A., Polo, J. y Giner, L. (2011). Burnout risk in medical students in Spain using the Maslach Burnout Inventory-Student Survey. International Archives of Occupational and Environmental Health, 84(4), 453-459. https:// doi.org/10.1007/s00420-011-0623-x

Gallagher, E.N. y Vella-Brodrick, D.A. (2008). Social support and emotional intelligence as predictors of subjective well-being. Personality and Individual Differences, 44, 1551-1561. https://doi.org/10.1016/ j.paid.2008.01.011

Gilman, R. y Huebner, S. (2003). A review of life satisfaction research with children and adolescents. School Psychology Quarterly, 18, 192-205. https:// psycnet.apa.org/doi/10.1521/scpq.18.2.192.21858

Goldbeck, L., Schmitz, T.G., Besier, T., Herschbach, P. y
Henrich, G. (2007). Life satisfaction during adolescence. Quality of Life Research, 16, 969-979. https://doi.org/10.1007/s11136-007-9205-5

Gutiérrez-Moret, M., Ibáñez-Martínez, R., Aguilar-Moya, R. y Vidal-Infer, A. (2016). Assessment of emotional intelligence in a sample of prospective secondary education teachers. Journal of Education for Teaching, 42(2), 123-134. https:// doi.org/10.1080/02607476.2016.1143144

Harrod, N.R. y Scheer, S.D. (2005). An exploration of adolescent emotional intelligence in relation to demographic characteristics. Adolescence, 40(159), 503512. Recuperado de https://search.proquest.com/ docview/195935644? accountid $=14555$

Hayes, A.F. (2013). Introduction to Mediation, Moderation, and Conditional Process Analysis: A Regression-based Approach. The Guilford Press.

Hogan, M., Parker, J., Wiener, J., Watters, C., Wood, L. y Oke, A. (2010). Academic success in adolescence: Relationships among verbal IQ, social support and emotional intelligence. Australian Journal of Psychology, 62(1), 30-41. https:// doi.org/10.1080/00049530903312881

Huebner, E.S. (2004). Research on assessment of life satisfaction of children and adolescents. Social Indicators Research, 66, 3-33. https://doi.org/10.1023/ B:SOCI.0000007497.57754.e3

Huebner, E.S., Drane J.W. y Valois R.F. (2000). Levels and demographic correlates of adolescent life satisfaction reports. School Psychology International, 21, 281-292. https://doi.org/10.1177\% 2F0143034300213005

Karasek, R.A. y Theorell, T. (1990). Healthy Work: Stress, Productivity and the Reconstruction of Working Life. Basic Books.

Kong, F., Zhao, J. y You, X. (2012). Social support mediates the impact of emotional intelligence on mental distress and life satisfaction in Chinese young adults. Personality and Individual Differences 53, 513-517. https://psycnet.apa.org/doi/10.1016/ j.paid.2012.04.021

Liau, A.K., Liau, A.W.L., Teoh, G.B.S. y Liau, M.T.L. (2003) The case for emotional literacy: The influence of emotional intelligence on problem behaviours in malaysian secondary school students. Journal of Moral Education, 32(1), 51-66. https:// doi.org/10.1080/0305724022000073338

Lopez-Zafra, E., Ramos-Álvarez, M.M., El Ghoudani, K., Luque-Reca, O., Augusto-Landa, J.M., Zarhbouch, 
B., Alaoui, S., Cortés-Denia, D. y Pulido-Martos, M. (2019). Social support and emotional intelligence as protective resources for well-being in Moroccan adolescents. Frontiers in Psychology, 10, 1529. https://doi.org/10.3389/fpsyg.2019.01529

Martins, A., Ramalho, N. y Morin, E. (2010). A comprehensive meta-analysis of the relationship between emotional intelligence and health. Personality and Individual Differences 49, 554-564. https:// doi.org/10.1016/j.paid.2010.05.029

Martínez, I. y Salanova, M. (2003). Niveles de burnout y engagement en estudiantes universitarios. Relación con el desempeño y desarrollo profesional. Revista de Educación, 330, 361-384.

Matud, M.P. (2004). Gender differences in stress and coping styles. Personality and Individual Differences, 37(7), 1401-1415. https://doi.org/10.1016/ j.paid.2004.01.010

Mayer, J.D. y Salovey, P. (1997). What is Emotional Intelligence? En P. Salovey y D. Sluyter (Eds.), Emotional Development and Emotional Intelligence. Implications for Educators (pp. 3-31). Basic Books.

Mayer, J.D., Roberts, R.D. y Barsade, S.G. (2008). Human abilities: Emotional intelligence. Annual Review Psychology, 59, 507-536. https://doi.org/10.1146/ annurev.psych.59.103006.093646

Montes-Berjes, B. y Augusto-Landa, J.M. (2007). Exploring the relationship between perceived emotional intelligence, coping, social support and mental health in nursing students. Psychiatric and Mental Health Nursing, 14(2), 163-171. https:// doi.org/10.1111/j.1365-2850.2007.01059.x

Palmer, B.R., Donaldson, C. y Stough, C. (2002). Emotional intelligence and life satisfaction. Personality and Individual Differences, 33, 1091-1100. https:// doi.org/10.1016/S0191-8869(01)00215-X

Palmer, B.R., Gignac, G., Manocha, R. y Stough, C. (2005). A psychometric evaluation of the MayerSalovey-Caruso Emotional Intelligence Test version 2.0. Intelligence, 33, 285-305. https:// doi.org/10.1016/j.intell.2004.11.003

Pandey, R. y Tripathi, A.N. (2004). Development of emotional intelligence: Some preliminary observations. Psychological Studies, 49, 147-150.

Pena, M., Rey, L. y Extremera, N. (2012). Life satisfaction and engagement in elementary and primary educators: Differences in emotional intelligence and gender. Revista de Psicodidáctica, 17(2), 341-360. Recuperado de https:/www.redalyc.org/articulo.oa?

\section{$\mathrm{id}=175 / 17523128006$}

Pérez-Fuentes, M.C., Molero-Jurado, M.M., SimónMárquez, M.M., Oropesa-Ruiz, N.F. y GázquezLinares, J.J. (2020). Validation of the Maslach Burnout Inventory-Student Survey in Spanish adolescents. Psicothema 32(3), 444-451. https:// doi.org/10.7334/psicothema2019.373

Peterson, K., Malouff, J. y Thorsteinsson, E.B. (2011). A meta-analytic investigation of emotional intelligence and alcohol involvement. Substance Use \& Misuse, 46(14), 1726-1733. http:// dx.doi.org/10.3109/10826084.2011.618962

Petrides, K.V., Frederickson, N. y Furnham, A. (2004). The role of trait emotional intelligence in academic performance and deviant behavior at school. Personality and Individual Differences, 36, 277-293. https:// doi.org/10.1016/S0191-8869(03)00084-9

Petrides, K., Pita, R. y Kokkinaki, F. (2007). The location of trait emotional intelligence in personality factor space. British Journal of Psychology, 98, 273-289. https://doi.org/10.1348/000712606X120618

Pinquart, M. y Sorensen, S. (2001). Gender differences in self-concept and psychological well-being in old age: A meta-analysis. Journal of Gerontology, 56 (4), 195-216. https://doi.org/10.1093/ geronb/56.4.P195

Purvanova, R.K. y Muros, J.P. (2010). Gender differences in burnout: A meta-analysis. Journal of Vocational Behavior, 77, 168-185. https://doi.org/10.1016/ j.jvb.2010.04.006

Ramos-Díaz, E., Rodríguez-Fernández, A., Axpe, I. y Ferrara, M. (2019). Perceived emotional intelligence and life satisfaction among adolescent students: The mediating role of resilience. Journal of Happiness Studies, 20, 2489-2506 (2019). https:// doi.org/10.1007/s10902-018-0058-0

Riaz, Z., Shahzad, S., y Ansari, K. (2009). Trait emotional intelligence as a determinant of life satisfaction and subjective happiness. Pakistan Journal of Psychology, 40(1). Recuperado de https:// search.proquest.com/docview/968415592? accountid $=1455$

Rico, D.G. (2009). Prevalencia del síndrome de burnout en estudiantes de nivel medio superior. Investigación Educativa Duranguense, 11, 29-38.

Rodríguez-Villalobos, J.M., Benavides, E.V., Ornelas, M. y Jurado, P.J. (2019). El burnout académico percibido en universitarios: comparaciones por género. Formación Universitaria, 12(5), 23-30. http:// 
dx.doi.org/10.4067/S0718-50062019000500023

Ryff, C. (1989). Happiness is everything, or is it? Explorations on the meaning of psychological well-being. Journal of Personality and Social Psychology, 57, 1069-1081.

Saklofske, D.H., Austin, E.J. y Minski, R.S. (2003). Factor structure and validity of a trait emotional intelligence measure. Personality and Individual Differences, 34, 707-721. https://doi.org/10.1016/S0191-8869 (02)00056-9

Saklofske, D.H., Austin, E.J., Mastoras, S.M., Beaton, L. y Osborne, S.E. (2012). Relationships of personality, affect, emotional intelligence and coping with student stress and academic success: Different patterns of association for stress and success. Learning and Individual Differences, 22(2), 251-257. https:// doi.org/10.1016/j.lindif.2011.02.010

Salavera, C., Usán, P. y Jarie, L. (2017). Emotional intelligence and social skills on self-efficacy in secondary education students. Are there gender differences? Journal of Adolescence, 60, 39-46. http:// dx.doi.org/10.1016/j.adolescence.2017.07.009

Salguero, J.M., Extremera, N., Cabello, R. y FernándezBerrocal, P. (2015). If you have high Emotional Intelligence (EI), you must trust in your abilities: The interaction effect of ability EI and perceived EI on depression in women. Journal of Psychoeducational Assessment, 33(1), 46-56. https:// doi.org/10.1177\%2F0734282914550384

Salovey, P, Stroud, L.R., Woolery, A y Epel, E.S. (2002). Perceived emotional intelligence, stress reactivity, and symptom reports: Further explorations using the Trait Meta-Mood Scale. Psychology and Health, 17 (5), 611-627. https:// doi.org/10.1080/08870440290025812

Sánchez-Álvarez, N., Extremera, N. y Fernández-Berrocal, P. (2016). The relation between emotional intelligence and subjective well-being: A meta-analytic investigation. The Journal of Positive Psychology, 11(3), 276-285. https:// doi.org/10.1080/17439760.2015.1058968

Sánchez-Álvarez, N., Berrios, M.P. y Extremera, N. (2020). A meta-analysis of the relationship between emotional intelligence and academic performance in secondary education: A multi-stream comparison. Frontiers in Psychology, 11, 1517. https:// doi.org/10.3389/fpsyg.2020.01517

Schaufeli, W.B., Martinez, M.I., Pinto, A.M., Salanova, M. y Bakker, A.B. (2002). Burnout and engagement in university students: A cross-national study. Journal of Cross-Cultural Psychology, 33 (5), 464-481. https://doi.org/10.1177\%2F0022022102033005003

Schneider, T.R., Lyons, J.B. y Khazon, S. (2013). Emotional intelligence and resilience. Personality and Individual Differences, 55(8), 909-914. https:// doi.org/10.1016/j.paid.2013.07.460

Shields, S.A. (2002). Speaking From the Heart: Gender and the Social Meaning of Emotion. Cambridge University Press.

Seligman, M. (2018). PERMA and the building blocks of well-being. The Journal of Positive Psychology, 13 (4), 333-335. https:// doi.org/10.1080/17439760.2018.1437466

Silveri, M.M., Tzilos, G.K., Pimentel, P.J. y YurgelunTodd, D.A. (2004). Trajectories of adolescent emotional and cognitive development: Effects of sex and risk for drug use. Annals of the New York Academy of Sciences, 1021(1), 363-370.

Trinidad, D.R., Unger, J.B., Chou, C.P. y Johnson, C.A. (2003). The protective association of emotional intelligence with psychosocial smoking risk factors for adolescents. Personality and Individual Differences 36, 945-954. https://doi.org/10.1016/S0191-8869 (03)00163-6

Uçak, E. y Yildirim, L. (2020). Investigation of the level of secondary school students' emotional intelligence in terms of different variables. International Journal of Psychology and Educational Studies, 7(1), 182-207. Recuperado de https://search.proquest.com/ docview $/ 2396837007$ ? accountid $=14555$

Usán-Supervía, P., Salavera-Bordás, C. y Mejías-Abad, J.J. (2020). Relaciones entre la inteligencia emocional, el burnout académico y el rendimiento en adolescentes escolares. Revista CES Psicología, 13(1), 125 -139. http://dx.doi.org/10.21615/cesp.13.1.8

Valadez-Sierra, M.D., Borges del Rosal, M.A., RuvalcabaRomero, N., Villegas, K. y Lorenzo, M. (2013). Emotional intelligence and its relationship with gender, academic performance and intellectual abilities of undergraduates. Electronic Journal of Research in Educational Psychology, 11(2), 395-412. http:// dx.doi.org/10.14204/ejrep.30.12204

Van Rooy, D.L., Alonso, A. y Viswesvaran, C. (2005). Group differences in emotional intelligence scores: Theoretical and practical implications. Personality and Individual Differences, 38, 689-700. https:// doi.org/10.1016/j.paid.2004.05.023

Veenhoven, R. (1996). Developments in satisfaction re- 
search. Social Indicators Research, 37, 1-46. https:// doi.org/10.1007/BF00300268

Vesely, A.K., Saklofske, D.H. y Leschied, A.D. (2013). Teachers-the vital resource: The contribution of emotional intelligence to teacher efficacy and wellbeing. Canadian Journal of School Psychology, 28
(1), 71-89.
https://doi.org/10.1177\%

2F0829573512468855

Whitman, D.S., Van Rooy, D.L., Viswesvaran, C. y Kraus, E. (2009). Testing the second-order factor structure and measurement equivalence of the Wong and Law Emotional Intelligence Scale across gender and ethnicity. Educational and Psychological Measurement, 69(6), 1059-1074. https://doi.org/10.1177\% 2F0013164409344498

Wong, C. y Law, D.S. (2002). The effects of leader and follower emotional intelligence on performance and attitude: An exploratory study. Leadership Quarterly, 13, 243-274. https://doi.org/10.1016/S10489843(02)00099-1 